\title{
Erratum: Economic growth, distribution policy and other factors: key elements in poverty alleviation
}

Silin Zhu, Zhiyan Liu and Yian Zhu

Original article: E3S Web of Conferences 253, 01042 (2021), https://doi.org/10.1051/e3sconf/202125301042

The second author of the article, namely Rishita Nandagiri, has been mistakenly included in the list of authors. It should be removed and included in the acknowledgements.

The authors, the guest editors and the Publisher agreed with the correction. 